\title{
BUILDING A SYNCHROTRON ONTOLOGY: AN ANALYSIS OF A SYNCHROTRON CONTROL SYSTEM IN A COLLABORATIVE ENVIRONMENT
}

\footnotetext{
Abstract

This paper presents research on building a synchrotron ontology using analysis of a synchrotron control system and the aspects of collaborative ontology engineering. It includes a general description of the studied domain and the method used to develop a synchrotron domain ontology. The ontology is being created on the basis of a Solaris synchrotron control system in cooperation with the synchrotron facilities belonging to the Tango community. The first Polish synchrotron radiation facility Solaris is located at Jagiellonian University's Third Campus in Krakow, Poland. Synchrotron is an unique source of electromagnetic radiation known as synchrotron radiation. This paper discusses the impact of the Solaris control system on the building of a synchrotron ontology. It also includes the main assumptions relating to the collaborative knowledge acquired for this domain.

The synchrotron ontology will support the optimization of existing control systems and the development of a new synchrotron control system based on Tango controls or other technologies in a consistent manner. Using the same general assumptions and terms, this could be later used for integration and data sharing purposes. The synchrotron ontology can facilitate interoperation by the integration of information from different sources from one or many synchrotron control systems and integrate different parts of the controls systems that provide analogical or similar services. It can also be used to support the translation between different representations, especially regarding particular devices. Knowledge sharing and reuse is a big challenge in complex, distributed systems where the knowledge required is very specialized for different sets of functionalities or subsystems. Regarding synchrotron systems, many specialists must provide their support so the IT specialists are able to develop and maintain a control system. In this case, the synchrotron ontology can be a guideline for knowledge sharing and reuse.

Keywords synchrotron ontology, Tango control system, building ontologies

Citation Computer Science 18 (1) 2017: 53-69
} 


\section{Introduction}

\subsection{Ontologies and ontology engineering}

Ontologies and ontology engineering have become very popular research topics (especially in the science and technology areas) and are increasingly being used not only in Artificial Intelligence but also in other disciplines of computer science [33]. In this field, ontologies are used in applications related to knowledge management and representation, e-commerce, language engineering, information retrieval and extraction, database design and integration, and the Semantic Web [29].

A lot of works have been presented [20] referring to all aspects of ontology engineering and concerning ontology development, the ontology life cycle, the methodologies for building ontologies, and the tools and languages that apply to them. An overview of the basic concepts and issues of ontological engineering are accurately described in [20].

Ontologies provide a number of useful features for intelligent systems, the most important being vocabulary, taxonomy, and knowledge sharing and reuse [14, 22, 24, $29,30]$. A taxonomy is a hierarchical classification of all entities within a domain, and it is represented in a machine-readable and processable form. An ontology cannot be defined as a taxonomy itself, as it is a full specification of a domain. Another feature is the vocabulary that refers to the terms in a described domain. Each term has unambiguous interpretation. Ontologies provide logical statements that specify what the terms are and how they are related; they also provide rules for combining the terms and the relationships between them. The major feature of ontologies is knowledge sharing and knowledge reuse by applications and intelligent agents. An ontology provides a full specification of a domain and contains a description of concepts and their relationships that can be shared and reused among intelligent systems.

\subsection{Synchrotron}

A synchrotron is a device in which electrons are accelerated to a very high energy in order to produce electromagnetic radiation of high intensity and wide range of energy [21]. The production of synchrotron radiation is a complex process that is possible due to the cooperation of a great number of devices implementing a set of specific tasks. Experts with knowledge in a given field are responsible for the supervision of individual devices. Members of the synchrotron team are specialists from various disciplines, such as physics, mechanics, electronics, and computer science.

The most important element of the synchrotron is an accelerator used to accelerate subatomic particles. Synchrotron Solaris, which is the first synchrotron in Poland, consists of two types of accelerators: a linear accelerator called Injector and a storage ring (wherein the electron beam achieves energy up to $1.5 \mathrm{GeV}$ ) [10]. The linear accelerator is made up of a preinjector - an electron gun, which is a source of electrons, and six straight sections called linac responsible for accelerating the electron beam energy to approx. $600 \mathrm{MeV}$. The storage ring consists of dipole and quadrupole mag- 
nets, whose task is to appropriate control electron beam [40]. The beam produced and accelerated in the linac is introduced through a transfer line to the storage ring located above. The task of the storage ring is to maintain circulating electron packets of a certain energy for as long as possible [11]. The electrons circulating in the storage ring produce synchrotron radiation, which goes to the measuring line or laboratories situated in the synchrotron hall. The description above reflects the life-cycle of electrons from generation to synchrotron radiation.

A synchrotron consists of numbers of devices that must be synchronized and controlled in order to maintain the electron beam and produce synchrotron radiation. For managing all devices, a distributed control system provides communication and data exchange between the devices and enables users to monitor the entire process. The synchrotron control system is an intelligent system that has the capacity to gather and analyze data and adapt according to the current data collected from different devices as well as data entered by users. The control system also enables remote monitoring and management. Above features determine the need to implement the full specification of all concepts, related activities and their relationships, to properly control and manage the synchrotron.

\subsection{Motivation}

Referring to Gruber's article, one of the reasons for building ontologies is to share a common understanding of the structure of information among people or software agents [22]. The main goal of the project is to build a synchrotron ontology that defines the concepts and relationships of the synchrotron device as well as activities related to the synchrotron operation in order to enable knowledge sharing and reuse it in a collaborative environment. At this point, the collaborative environment means cooperating people who are experts in a particular subject area and a distributed control system responsible for managing the synchrotron device. The development of the ontology is also connected with the technology used to build synchrotron control systems called Tango controls [34]. The Tango control system is a solution dedicated, inter alia, for building distributed, object-oriented control systems, and it is used by the group of synchrotron facilities as a system for controlling the production of radiation.

The motivation to build a synchrotron ontology is the optimization and development of a reusable architecture for synchrotron control systems to be used for building systems based on Tango controls or other technologies. In general, the optimalization process involves identifying all goals and processes that are used to reach them and simplifying the process to reach the same goals. In this case, the work done during the building of the synchrotron ontology is the first step of optimalization of the synchrotron control system. Other applications of synchrotron ontology are interoperation, education, and modeling $[17,28]$. The synchrotron ontology can facilitate interoperation by the integration of information from different sources of one or many synchrotron control systems. It can also be used to support the translation 
between different representations, especially regarding particular devices. The synchrotron ontology will be a good source of reference and enable people from different specializations to learn more about the synchrotron domain. One of the most important applications of synchrotron ontology is modeling. As the synchrotron ontology represents important reusable building blocks, it can be used in applications related to the synchrotron control system, like the synchrotron system itself. Such predefined blocks could be included in applications as pre-developed modules [17].

The rest of the paper is organized as follows: Section 2 presents the state of knowledge and existing projects about synchrotron ontologies. Section 3 explains the difference between application and domain ontology and briefly describes the synchrotron ontology and the Tango control system. It also discusses their impact on the development of a method for creating the synchrotron ontology. Section 4 presents the ontology building approaches of the application and domain synchrotron ontology. In this section, the first version of the synchrotron domain ontology is described. Section 5 reviews all techniques used for evaluating the synchrotron ontology. Finally, Section 6 provides the conclusion of this paper, including potential future research.

\section{Related work}

\subsection{Web repositories and libraries of ontologies}

In order to verify the state of knowledge and existing projects regarding synchrotron ontology as well as related ontologies that could be merged with the synchrotron ontology, the following list of available libraries, repositories, and other sources has been researched $[2-7,9]$. In order to prepare a reliable source of reference, we focused primarily on a search of the scientific literature [15], then we researched all founded repositories and libraries. The presented libraries and repositories were selected based on their frequency of use in the scientific literature and number of resources. The results are presented in Table 1, which consists of the following columns: the source name, a short description of the source, the type of domain (where the value "General" indicates that there are no restrictions on the content according to the domain), and two distinct descriptions of the results of the research.

There is much research on ontologies or the implementation of them that support different areas related to accelerators and physics (e.g., [12, 41]); however, none of them describe the accelerator machine itself, nor all of the relationships between devices that need to cooperate to produce electromagnetic radiation. In this field, no work has been found.

The results are inconclusive. There is no synchrotron ontology or any project that applies its concepts. One of the steps of building an ontology is merging it with existing ontologies that describe related concepts. We didn't find ontologies that can be merged with a synchrotron ontology. 
Table 1

List of repositories and search engines used to verify the state-of-the-art of synchrotron ontology $[2-7,9]$.

\begin{tabular}{|l|l|l|l|l|}
\hline Source & Description & Domain & $\begin{array}{l}\text { Synchrotron } \\
\text { ontology }\end{array}$ & $\begin{array}{l}\text { Related } \\
\text { ontologies }\end{array}$ \\
\hline Cupboard[2] & ontology repository & General & No & No \\
\hline ODP[5] & $\begin{array}{l}\text { repository for design patterns and } \\
\text { ontology modules following the } \\
\text { patterns }\end{array}$ & General & No & No \\
\hline OntoSelect[6] & $\begin{array}{l}\text { ontology library and ontology } \\
\text { selection service }\end{array}$ & General & No & No \\
\hline DAML[3] & $\begin{array}{l}\text { ontology library includes records of } \\
\text { ontologies from around the Web }\end{array}$ & General & No & No \\
\hline $\begin{array}{l}\text { Protégé } \\
\text { Lntology }\end{array}$ & $\left.\begin{array}{l}\text { list of user-provided ontologies } \\
\text { hosted on the Protégé project wiki }\end{array}\right]$ & General & No & No \\
\hline Swoogle[9] & Semantic Web Search Engine & General & No & No \\
\hline Falcons[4] & Semantic Web Search Engine & General & No & No \\
\hline
\end{tabular}

The research shows that there is no synchrotron ontology defined so far and there are no official projects that aim to build a synchrotron ontology or other ontologies that would describe a domain related to the synchrotron domain.

\subsection{ScienceWISE project}

The ScienceWISE project's aim is to develop an online knowledge base that is integrated into the ArXiv.org, which is an open access to e-prints in Physics, Mathematics, Computer Science, Quantitative Biology, Quantitative Finance, and Statistics $[1,8]$. The ScienceWISE Web tool enables users to generate professional specific ontologies, add concepts as well as the logical relationships between them, manage several definitions of the same concept, and access a mechanism for viewing rankings made by the scientific community in order to identify the best definitions.

The physics ontologies provided by the ScienceWISE project do not directly represent the synchrotron domain, but they do show general physics concepts as distinct science fields. The ontologies referring to the physics domain describe its concepts, taking into account several different categories. Subcategories regarding concepts that could correspond with those used in the synchrotron ontology are General Physics (a subcategory of the Formalism Category that provides general physics concepts from physics disciplines) and Measuring Devices (which provides knowledge about the various devices used in physics for measuring different physical features). Some of the devices presented in this category are used in synchrotrons. Selected concepts, groups of concepts, and their relationships to the above-mentioned categories can be used while creating the synchrotron ontology; these are also taken into consideration. 


\section{Synchrotron ontology}

\subsection{Synchrotron as application and domain ontology}

A synchrotron is used to accelerate electrons that produce light (which is actually an electromagnetic radiation of a wide spectral range from infrared to X-ray radiation). Synchrotrons differ in the scope of their construction, applied technology, and devices. Because of the large variability in applied technologies, the vocabulary used in particular synchrotron systems is very diverse.

According to the classification of ontologies introduced by Guarino [25], an application ontology is most specific (taking into account the level of dependence on a particular task or point of view), while a domain ontology describes the vocabulary related to a generic domain or task. Another categorization described by van Heijst [26] in which the division criteria was the subject of the conceptualization also marks out application and domain ontologies. An application ontology is applicationdependent and contains all of the definitions needed to model the knowledge required for a particular application. Application ontologies often extend and specialize the vocabulary of domain ontologies [20], and this relationship is going to be used when building the synchrotron domain ontology (see Figure 1).

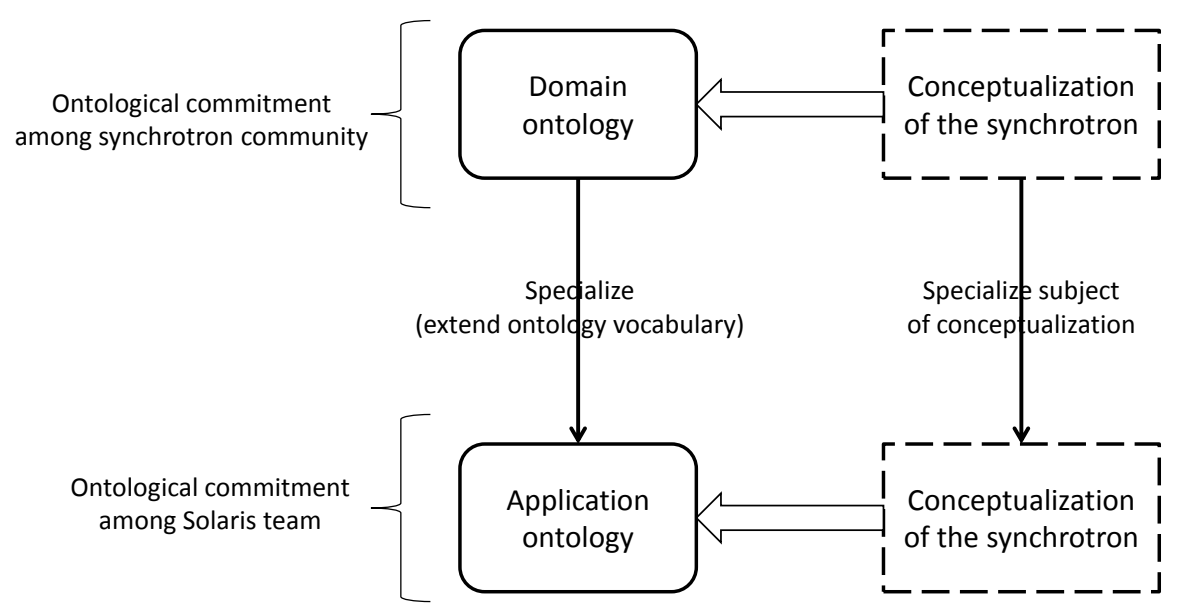

Figure 1. Relationship between application and domain ontologies of a synchrotron.

The main difference between a synchrotron application ontology and a domain ontology is the reusability of the latter. Reusability of the synchrotron domain ontology implies the possibility to use it for different synchrotrons. Ontology usage refers to the software, but also to other aspects such as activities, theories, and principles governing that domain. The aim of this work is to build a domain ontology at such a level of abstraction to give freedom to specialize and instantiate the ontol- 
ogy as required; in particular, to allow design reuse and optimization of the existing synchrotron control systems.

\subsection{Domain conceptualization}

A conceptualization was defined by Genesereth and Nilsson as a structure $\langle D, R\rangle$, where $D$ is a domain and $R$ is a set of relationships on $D$ [18]. Based on this definition, Gruber defined an ontology as an explicit specification of a conceptualization [22]. To independently construct relationships of a state of affairs, Guarino proposed extending the definition of conceptualization $C=\langle D, W, S\rangle$, where $D$ is a domain, $W$ is a set of possible worlds (states of affairs of such domain), and $S$ is a set of conceptual relationships on the domain space $\langle D, W\rangle[25]$. On the basis of these theories, knowledge of a synchrotron Solaris domain should be represented in a formalized way by a set of concepts and relationships, taking into account possible states of affairs. The state of affairs of the Solaris synchrotron domain is strictly related to the synchrotron radiation production process that is managed by the Tango control system. The Tango control system is an open-source, object-oriented, and distributed system for controlling hardware and software [35]. It is based on the concept of TDSOM (Tango Device Server Object Model), where all controlled devices belong to classes and are represented by objects. The Tango system consists of clients and device servers that provide services to one or more clients. Device servers are designed to allow users to read and write data from all devices in the control system [34]. The system is based on CORBA technology (which provides synchronous and asynchronous communication) and zeroMQ technology (which allows for event- driven communication). These communication technologies enable the integration of objects working in heterogeneous computer systems [13]. Due to the relationship between the synchrotron domain and the synchrotron control system, an analysis of the Solaris Tango control system was one of the first tasks needed to gain basic knowledge about the synchrotron domain and identify the state of affairs. The developed object model of the Solaris synchrotron control system is the basis for concepts and relationships as well as the extraction of necessary vocabulary.

\subsection{Achieving ontological commitment}

One of the basic problems to overcome during the building of ontologies is to achieve ontological commitment within the contributing community and to ensure interoperability between systems and humans. Referring to the Gruber and Olsen definition, ontological commitment is an agreement to use the shared vocabulary in a coherent and consistent manner [23]. This is a connection between the ontology vocabulary and the meaning of the terms of such vocabulary [25]. In the creation of a synchrotron ontology, different perspectives have to be taken into account; therefore, a collaborative ontology building approach is going to be used. The methodology or methods to be used depend on the conditions of the project and the analysis performed during the earlier stages. 
As an ontology is a shared and a common understanding of some domain [16], the conducted research focused on developing an ontology in cooperation with the synchrotron community whilst keeping an agreement on the content of synchrotron ontology. The ontological commitment should be verified by taking into account two aspects: a consensus between domain experts belonging to different disciplines (responsible for different subsystems) and a consensus between synchrotron facilities.

The ontology development process should correspond with distributes community of interests, which is distributed in a sense of distinct knowledge, responsibilities, and geographical dispersion. The differences between domain and application ontologies impose a range of ontological commitment for the synchrotron domain. To achieve a shared and common taxonomy of the domain ontology, we should ensure a consensus between domain experts from different facilities. To achieve ontological commitment for the synchrotron application ontology, the group of domain experts should be restricted to experts from the Solaris facility.

\section{Ontology development process}

\subsection{Domain-application ontology building approach}

In the literature, there are lots of methodologies and methods presented for the purpose of building ontologies from scratch [14, 16, 31, 33]. The relationship between methodologies and methods is that a methodology is a series of methods and techniques while a method is a general procedure that can be applied by using specific techniques [32].

The work includes building a synchrotron domain ontology based on the synchrotron Solaris control system using a collaborative ontology developing process. It is a target ontology that should fulfill the project goals.

As mentioned in previous sections, building a synchrotron domain ontology requires collaboration within the synchrotron community. After review of collaborative methodologies, we have decided to elaborate our collaborative domain ontology design approach based on the collaborative methodology proposed by Holsapple and Joshih [27] as well as elements of Uschold and King's method [37]. The reasons for choosing these approaches are as follows: it enables both domain and application ontology building in a collaborative way, it is straightforward and accessible to a large community of domain experts, and it enables us to achieve ontological commitment within the contributing community. An important aspect of choosing a particular methodology is that the control system is already implemented and the business and system analysis are finished.

Holsapple and Joshih divide the engineering process into four phases: preparation, anchoring, iterative improvement, and application. The preparation phase determines boundary conditions, design criteria, and ontology evaluation standards. In this step, we additionally identify purposes for building ontology [37], specific applications of the ontology. The goal of the anchoring phase is to develop an initial 
ontology by merging existing ontologies. Most of the methodologies for building ontologies assume that, at some point of the building process, there is an adaptation of existing ontologies in order to improve the development of the target ontology [20]. Preliminary research on existing ontologies and libraries that could be used for building a synchrotron ontology failed to find taxonomies that match our requirements and that could be integrated with a synchrotron ontology. Therefore, a synchrotron ontology will be built from scratch; in our approach, this will occur during the anchoring phase. To capture a synchrotron ontology, a top-down strategy is used [37]. The next phase (iterative improvement) adjusts and extends the anchor ontology using a Delphi-like approach to incrementally improved the shared and reusable domain ontology. The last application phase shows the use of a synchrotron ontology in a specific context. In our case it is a synchrotron application ontology. Therefore, the last phase covers the whole application ontology building process.

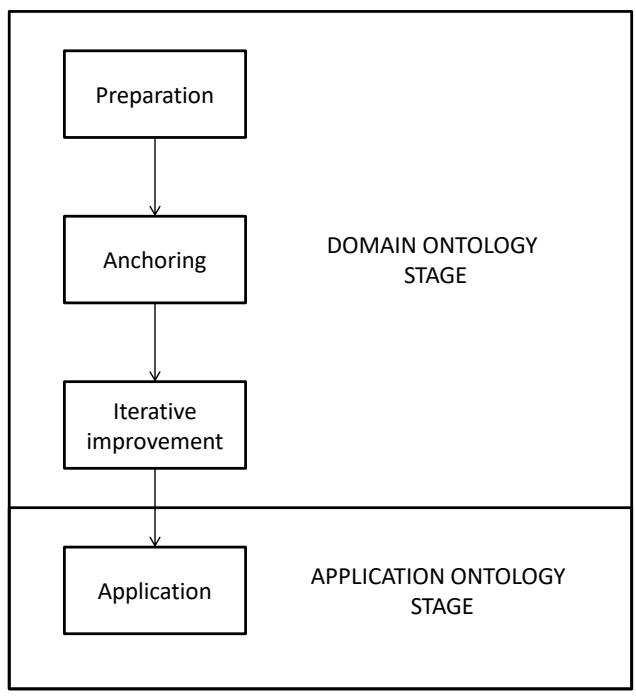

Figure 2. Domain-application ontology building approach.

A collaborative ontology developing process starts with an analysis of the domain to be captured by the ontology as well as the requirements imposed by the ontologybased application [31]. A domain ontology will be designed upon the analysis of the Solaris synchrotron control system as well as gathering knowledge from synchrotron community experts. In the preparation and anchoring phases, the domain expert community is restricted to Solaris synchrotron experts, while in the iterative improvement phase, the expert group is extended to the synchrotron community.

The result of mapping the modified Holsapple and Joshih methodology to our two-stage approach for building domain and application ontology is that the preparation, anchoring, and iterative improvement phases refer to a domain ontology, and the application phase refers to an application ontology. This process is shown in Figure 2. 
The application-based ontology will be designed on the basis of the synchrotron domain ontology (upon analysis of the Solaris synchrotron control system as well as gathering knowledge from Solaris experts). During this process, the ontology vocabulary will be specified in order to extend the concepts and relationships. The application-based ontology should consist of vocabulary referring to all devices and their possible states. As the control system is implemented, the ontology will be created using class diagrams that show concepts and relationships that belong to the domain. The method or methodology for building the application ontology has yet to be developed.

The two-stage approach involving the entire ontology building process is shown in Table 2. In both stages, the Solaris control system analysis is the input of starting the collaborative ontology building process.

Table 2

The two-stage approach of ontology building process.

\begin{tabular}{|l|l|l|l|}
\hline Stage & $\begin{array}{l}\text { Building domain } \\
\text { ontology (Anchor } \\
\text { version) }\end{array}$ & $\begin{array}{l}\text { Building domain } \\
\text { ontology (Final } \\
\text { version) }\end{array}$ & $\begin{array}{l}\text { Building application } \\
\text { ontology }\end{array}$ \\
\hline Used method & $\begin{array}{l}\text { Holsapple and Joshih, } \\
\text { Uschold and King }\end{array}$ & $\begin{array}{l}\text { Holsapple and Joshih, } \\
\text { Uschold and King }\end{array}$ & In preparation \\
\hline $\begin{array}{l}\text { Domain } \\
\text { expert }\end{array}$ & Solaris experts & $\begin{array}{l}\text { Synchrotron community } \\
\text { experts }\end{array}$ & Solaris experts \\
\hline $\begin{array}{l}\text { Knowledge } \\
\text { engineer }\end{array}$ & author & $\begin{array}{l}\text { Synchrotron community } \\
\text { experts }\end{array}$ & author \\
\hline $\begin{array}{l}\text { Ontology } \\
\text { engineer }\end{array}$ & author & author & author \\
\hline $\begin{array}{l}\text { Input } \\
\text { control system }\end{array}$ & $\begin{array}{l}\text { First version of } \\
\text { synchrotron domain } \\
\text { ontology in OWL }\end{array}$ & $\begin{array}{l}\text { Specification of Solaris } \\
\text { control system and } \\
\text { Synchrotron Solaris } \\
\text { application ontology in } \\
\text { OWL }\end{array}$ \\
\hline Output & $\begin{array}{l}\text { Anchor version of } \\
\text { synchrotron domain } \\
\text { ontology in OWL }\end{array}$ & $\begin{array}{l}\text { Synchrotron domain } \\
\text { ontology in OWL }\end{array}$ & $\begin{array}{l}\text { Synchrotron Solaris } \\
\text { application ontology in } \\
\text { OWL }\end{array}$ \\
\hline Used tool & \multicolumn{2}{|c|}{ Protégé } \\
\hline
\end{tabular}

In ontology engineering, we can distinguish three roles: domain experts, knowledge engineers, and ontology engineers [20]. Domain experts have knowledge about the domain, its concepts, and their relationships. The role of ontology engineers is to gain knowledge from domain experts to create a conceptual model of the domain that, in the next step, is presented by ontology engineers with the use of the appropriate representation language. The ontology engineering process is driven by engineers who gather requirements, implement these requirements, and test the resulting ontology. Each member of the community can play any of the roles. During the realization of this project, the role of knowledge engineer and ontology engineer will be connected 
and assigned to one person during the application ontology development process, and knowledge engineer and ontology engineer roles will be assigned to different persons while building the synchrotron domain ontology.

After analysis of the available tools that support the collaborative ontology development process, the tool that is going to be used to build our synchrotron ontology is Protégé. Protégé allows us to represent the ontology in OWL (Web Ontology Language). It has a user-friendly interface and modules that enable cooperation (annotation module, workflow module, or changes module [36]). After research on ontology representation languages in terms of their expressiveness and reasoning, the language chosen to implement the synchrotron ontology was OWL 2. OWL 2 is a computational logic-based language designed to represent complex knowledge about things and relationships between things [20]. OWL is designed for use by applications that need to process information. Its fundamental modeling components are classes, slots, facets, and instances, and it is the appropriate language to describe the synchrotron ontology referring to the object-oriented control system.

\subsection{First version of synchrotron domain ontology}

Using the control system specification (and in cooperation with Solaris domain experts), the first version of our synchrotron domain ontology has been created. While identifying key concepts and relationships in the synchrotron domain, the following assumptions were adopted: a synchrotron consists of hardware and software, and these two concepts need to be taken into account. In a synchrotron, we can distinguish elements (partitions) related to the synchrotron radiation production process.

The control system coordinates all of the elements of the synchrotron mentioned in Section 1.2. The structure and function of each synchrotron both determine the division of the control system into the following parts: Preinjector, Linear Accelerator, Transfer Line, and Storage Ring. This division reflects the life-cycle of electrons (from generation to synchrotron radiation) but does not reflect the services provided by specific devices, types of equipment, and the software that is part of the control system. Each partition performs its tasks through the cooperation of many devices (which are all controlled by the control system). Devices (objects) are the basis of the object-oriented system TANGO and create subsystems of the synchrotron control system. In different parts of the synchrotron, there are the same devices or different types of the same basic device having specific functions. Therefore, the subsystem can operate in different partitions, and the individual partitions carry out their tasks through the cooperation of many subsystems.

Base of the following relationships the general concepts has been prepared:

- Preinjector, Linear Accelerator (Linac), Transfer Line, Storage Ring - refer to the synchrotron device partitions and reflects the life-cycle of electrons,

- Software - refers to the synchrotron control system,

- Device - refers to the devices that are controlled by the synchrotron system and work in synchrotron partitions. 
The device concept has been additionally divided into a group of devices that should occur in all synchrotrons. We don't determine the exact technology that can be used, but the general vocabulary for devices that perform certain functions in the synchrotron:

- Magnet - magnet devices that maintain track the motion of electrons and their power,

- Cooling - devices responsible for maintaining the temperature of the individual components / devices in the desired range, preventing overheating,

- Diagnostic - devices used for the diagnosis of the beam that monitors the location and quality of the beam in the transfer line and storage ring,

- RF - devices responsible for the control units providing RF energy to accelerate the electrons,

- Optics - optics devices responsible for the optic diagnostic,

- Vacuum - devices responsible for maintaining the proper vacuum in the vacuum chamber in order to avoid the loss of an electron beam and prolong the life of the beam,

- PSS - devices from the Personal Safety System that are responsible for protecting people from synchrotron radiation.

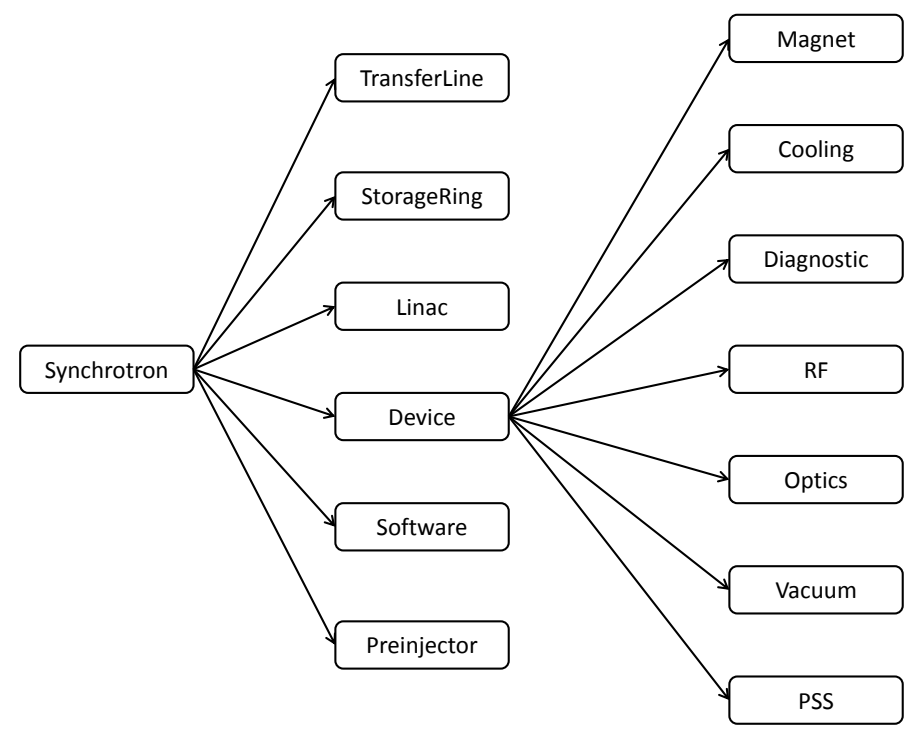

Figure 3. Domain synchrotron ontology.

The first (anchor) version of the domain ontology has been developed in the OWL DL sublanguage (which includes all OWL language constructs using Protégé). Figure 3 shows the result graph. The synchrotron is a base concept and is the parent class for Device, Software, and partition classes. We don't create the partition class 
but set all the partitions directly under the synchrotron, as partition word is not a common concept and does not bring additional knowledge.

The class axioms used for Device, Software, and partition classes are as follows:

\section{Device}

Device $\sqsubseteq$ Synchrotron

Device $\sqsubseteq \neg$ Software

DisjointUnion Linac Preinjector StorageRing TransferLine

\section{Software}

Software $\sqsubseteq$ Synchrotron

Software $\sqsubseteq \neg$ Device

Partition classes: Preinjector, Linac, TransferLine, StorageRing

partition class $\sqsubseteq$ Synchrotron

The first version of our synchrotron domain ontology is an input that describes the most basic and common concepts and relations between them. Based on this we have started the collaborative ontology building process in the synchrotron community.

\section{Ontology evaluation}

Ontologies are used in order to ensure a standard method for data exchange between systems and within the systems by various components in a consistent and shared way. Ontologies engineer artifacts that should be evaluated [38]. According to Gómez-Pérez [19], an ontology evaluation is the technical judgment of the content of the ontology with respect to a frame of reference such as requirement specifications, competency questions, or the real-world during each phase of their lifecycle. Ontology evaluation includes aspects of ontology verification (which refers to building the ontology correctly) and ontology validation aspects (which refer to whether the ontology definitions model the real world). Ontologies can be evaluated by taking into account quality criteria and ontology aspects. The quality criteria are: accuracy, adaptability, clarity, completeness, computational efficiency, conciseness, consistency, and organizational fitness [33].

The methods of evaluating ontologies are different and depend on aspects of each ontology. Ontology aspects are vocabulary, syntax, structure, semantics, representation, and context [33]. Each aspect has a different evaluation approach that could also be used in order to evaluate the synchrotron ontology.

The vocabulary of an ontology is the set of all names identified in the ontology by URIs (Uniform Resource Identifiers) or literals that should be evaluated (in terms of their naming convention and explicitness, in particular).

Ontologies can be described using different syntaxes. The syntax that is going to be used to present the synchrotron ontology is OWL 2. Taking into account this aspect, the synchrotron ontology should be transformable automatically from OWL 2 
into other syntaxes (like RDF/XML), and such a transformation is going to be made and analyze.

The structure of each ontology can be represented by an RDF graph; this is one of the most-widely-used measurement aspects of ontologies. An ontology that is represented in OWL can also being mapped on the RDF graph representation. To evaluate aspects of a synchrotron ontology structure, both application and domain ontologies will be mapped on RDF graphs.

Another measure aspect is semantics. To evaluate this ontology aspect, normalization could be used. Normalization is used to transform the structure of an ontology to make the semantics explicit within the structure while keeping the semantics [39].

The representation aspect that link the relationship between the structure and the semantics will be evaluated by comparing the RDF graph with features of possible models that provide the ontology.

The context of the ontology refers to the features of the ontology when compared with the aspects of ontology environment; e.g., a different representation of the data within the ontology [33].

Both the application and domain synchrotron ontology will be evaluated, taking into account the above-mentioned aspects in order to optimize and achieve a good quality ontology that meets the reusable and shared requirements. The evaluation results will be presented in thesis.

\section{Conclusions and future work}

The thesis presented in this article is in its initial stage. However, the work that has been done within the framework of the thesis comprises research on existing methodologies for building, tools for developing, and languages for representing ontologies. The verification of existing ontologies shows that there is no ontology that would describe a synchrotron domain. Conducted studies and analysis of the Solaris control system resulted in the creation of a synchrotron development ontology process in which the application domain development process is developed entirely. The mentioned research gives a view on the state of art in the field of ontology engineering and collaborative ontology engineering.

The implementation of the project assumes the following stages: building a synchrotron domain ontology, presenting the ontology in OWL, constructing the first version of the synchrotron domain ontology in cooperation with the Solaris team, creating the method of the synchrotron domain ontology development process, implementing the method in order to create a synchrotron domain ontology in cooperation with the synchrotron community. Both application and domain ontologies will be evaluated, taking into account quality criteria and ontology aspects.

The aim of this project is to build a synchrotron domain ontology in order to perform a domain analysis to cover possibly the largest number of synchrotron facilities. The achieved results should enable the reuse of domain knowledge to integrate actual control systems and to implement future systems used in synchrotrons. 


\section{References}

[1] ArXiv.org an open access. http://arxiv.org/, accessed: 2016.01.24.

[2] Cupboard. http://cupboard.open.ac.uk:8081/cupboard-search/, accessed: 2016.01.20.

[3] DAML Ontology Library. http://www.daml.org/ontologies/, accessed: 2016.01.20.

[4] Falcons. http://ws.nju.edu.cn/falcons/objectsearch/index.jsp, accessed: 2016.01.20.

[5] Ontology Design Patterns. http://ontologydesignpatterns.org/wiki/Main Page, accessed: 2016.01.20.

[6] OntoSelect. http://olp.dfki.de/ontoselect/, accessed: 2016.01.20.

[7] Protege Ontology Library. http://protegewiki.stanford.edu/wiki/ Protege_Ontology_Library\#OWL_ontologies, accessed: 2016.01.20.

[8] ScienceWISE project. http://sciencewise.info/, accessed: 2016.01.24.

[9] Swoogle semantic web search. http://swoogle.umbc.edu/, accessed: 2016.01.20.

[10] Bartosik M., Bocchetta C., Borowiec P., Goryl P., Nietubyć R., Stankiewicz M.: Solaris National Synchrotron Radiation Centre, project progress, May2012. Radiation Physics and Chemistry, pp. 4-8, 2013.

[11] Bocchetta C., Goryl P., Królas K., Młynarczyk M., Stankiewicz M., Tracz P.: Project status of the Polish synchrotron radiation facility Solaris. IPAC 11, San Sebastian, THPC054, p. 3014, 2011.

[12] Carral D., Cheatham M., Dallmeier-Tiessen S., Herterich P., Hildreth M., Hitzler P., Krisnadhi A., Lassila-Perini K., Sexton-Kennedy E., Vardeman C., Watts G.: An Ontology Design Pattern for Particle Physics Analysis. http://ceur-ws. org/Vol-1461/WOP2015_pattern_abstract_5.pdf, accessed: 2016.07.26.

[13] Chaize J.M., Götz A., Klotz W.D., Meyer J., Perez M., Taurel E.: TANGO - an object oriented control system based on CORBA. http://www.tango-controls . org/media/filer_public/06/8e/068ea5a4-bc16-49a6-a202-a451ad45eca3/ tango_an_object_oriented_control_system_based_on_corba.pdf, accessed: 2016.09.18.

[14] Chandrasekaran B., Josephson J., Benjamins V.: What are ontologies, and why do we need them? IEEE Intelligent Systems, vol. 14, pp. 20-26, 1999.

[15] d'Aquin M., Noy N.: Where to publish and find ontologies? A survey of ontology libraries. Web Semantics: Science, Services and Agents on the World Wide Web, vol. 11, p. 96111, 2012.

[16] Fernández-López M., Gómez-Pérez A.: Overview and analysis of methodologies for building ontologies. The Knowledge Engineering Review, pp. 129-156, 2002.

[17] Fikes R.: Multi-use Ontologies, Stanford University. http://web.archive.org/ web/20080207010024/http://www.808multimedia.com/winnt/kernel.htm, accessed: 2015.09 .30 . 
[18] Genesereth M.R., Nilsson N.J.: Using explicit ontologies in KBS development. Logical foundations of artificial intelligence, Morgan Kaufmann Publishers, San Francisco, 1987.

[19] Gómez-Pérez A.: A Framework to Verify Knowledge Sharing Technology. Expert Systems with Application, vol. 11, pp. 519-529, 1996.

[20] Gómez-Pérez A., Fernández-López M., Corcho O.: Ontological Engineering with examples from the areas of Knowledge Management, e-Commerce and the Semantic Web. Springer, Berlin, 2004.

[21] Goryl P., Bocchetta C., Królas K., Młynarczyk M., Nietubyć R., Stankiewicz M., Tracz P., Walczak E., Wawrzyniak A.: Solaris project status and challenges. Proceedings of ICALEPCS2011, p. 411, 2011.

[22] Gruber T.: A translation approach to portable ontologies. Knowledge Acquisition, vol. 5, pp. 199-220, 1993.

[23] Gruber T.R., Olsen G.: An Ontology for Engineering Mathematics. In: J. Doyle, P. Torasso, E. Sandewall, eds., Principles of Knowledge Representation and Reasoning: Proceedings of the 4th International Conference (KR '94): Bonn, Germany: 1994, May, 24-27, The Morgan Kaufmann Series in Representation and Reasoning, pp. 258-269, Morgan Kaufmann Publishers, 1994.

[24] Guarino N.: Formal ontology, conceptual analysis and knowledge representation. International Journal of Human-Computer Studies, vol. 43, pp. 625-640, 1995.

[25] Guarino N.: Formal Ontology in Information Systems. 1st International Conference on Formal Ontology in Information Systems (FOIS 98), IOS Press, pp. 3-15, 1998.

[26] van Heijst G., Schreiber A., Wielinga B.: Using explicit ontologies in KBS development. International Journal of Human-Computer Studies, pp. 183-292, 1995.

[27] Holsapple C.W., Joshi K.D.: A collaborative approach to ontology design. Communications of the ACM, vol. 2, pp. 42-47, 2002.

[28] Jardim-Gonçalves R.J., Müller J.P., Mertins K., Zelm M.: Enterprise Interoperability II, New Challenges and Approaches. Spinning the Semantic Web: Bringing the World Wide Web to Its Full Potential, eds. D. Fensel, J. Hendler, H. Lieberman and W. Wahlster, Berlin, 2007.

[29] McGuinness D.: Ontologies come of age. Spinning the Semantic Web: Bringing the World Wide Web to Its Full Potential, eds. D. Fensel, J. Hendler, H. Lieberman, W. Wahlster, Boston, 2002.

[30] Schreiber G., Wielinga B., de Hoog R., Akkermans H., van de Velde W.: CommonKADS: A comprehensive methodology for KBS development. IEEE Expert, vol. 9, pp. 28-37, 1994.

[31] Simperl E., Luczak-Rosch M.: Collaborative ontology engineering: a survey. The Knowledge Engineering Review, pp. 101-131, 2013.

[32] Society I.C.: IEEE Standard Glossary of Software Engineering Terminology.

[33] Staab S., Studer R.: Handbook on Ontologies. Springer, Berlin, 2009. 
[34] TANGOTeam: The TANGO Control System Manual. Retrieved June 26, 2015. http://www.esrf.eu/computing/cs/tango/tango_doc/kernel_doc/ds_ prog/, accessed: 2016.09.18.

[35] Taurel E.: TANGO collaboration and kernel status. Retrieved June 26, 2015. http://plone.tango-controls.org/Documents/papers/icalepcs-2011/at_ managed_file.2011-10-12.4708123844, accessed: 2016.09.18.

[36] Tudorache T., Noy N., Tu S., Musen M.: Supporting Collaborative Ontology Development in Protégé. In The Semantic Web - ISWC 2008, Lecture Notes in Computer Science, vol. 5318, pp. 17-32, 2008.

[37] Uschold M., King M.: Towards a Methodology for Building Ontologies. IJCAI'95 Workshop on Basic Ontological Issues in Knowledge Sharing, Montreal, pp. 6.16.10, 1995.

[38] Vrandečić D.: Ontology Evaluation. Ph.D. thesis, Wirtschaftswissenschaften, Karlsruhe Institute of Technology, Germany, 2010.

[39] Vrandečić D., Sure Y.: How to design better ontology metrics. Proceedings of the 4th European Semantic Web Conference (ESWC 07), 2007.

[40] Wawrzyniak A., Bocchetta C., Leemann S., Thorin S.: Injector layout and beam injection into Solaris. IPAC 11, San Sebastian, THPC123, p. 3173, 2011.

[41] Yepes A., Rousseau B.: Towards an ontology based search mechanism for the EDMS at CERN. Proceedings of EPAC 2004, 2004.

\section{Affiliations}

\section{Julia Szota-Pachowicz}

National Synchrotron Radiation Centre SOLARIS, ul. Czerwone Maki 98, Krakow and Jagiellonian University, Faculty of Physics, Astronomy and Applied Computer Science, ul. prof. Stanisława Łojasiewicza 11, Krakow, julia.szota@doctoral.uj.edu.pl

Received: 2.02 .2016

Revised: 31.07 .2016

Accepted: 5.08 .2016 